\title{
New biomarker panel prognosticates patient survival
}

The newly published results of a phase III clinical trial reveal that circulating tumour cell (CTC) enumeration and lactate dehydrogenase (LDH) levels are an effective biomarker of castration-resistant prostate cancer (CRPC). These findings might enable better clinical decisionmaking and management of patients with this disease.

In a multicentre study, patients receiving treatment for CRPC (abiraterone plus prednisone or prednisone monotherapy) were also monitored for a range of blood biomarkers that included: CTCs, prostatespecific antigen, LDH, haemoglobin, albumin, and alkaline phosphatase. Cutoff points for each biomarker level were based upon upper or lower limits of 'normal' as reported by other sources, including FDA guidelines and published nomograms. Biomarker suitability was evaluated in line with the Prentice criteria.

After 12 weeks of treatment, CTC numbers alone and $\mathrm{LDH}$ alone were found to be the most effective markers for discrimination between high-risk patients and low-risk patients; however, individual use of either of these markers failed to eliminate the treatment effect on survival in the model used.

CTC number and LDH proved superior to any of the other biomarkers tested in terms of concordance with survival, either alone, or in combination with any two biomarkers. For this reason, $\mathrm{LDH}$ and CTC measurements were combined into a biomarker panel. Patients with $<5$ CTCs per $7.5 \mathrm{ml}$ of blood were classified as low-risk, and patients with $\geq 5$ CTCs with LDH $>250 \mathrm{U} / \mathrm{L}$ were classified as high-risk. Patients classified as low-risk, and those classified as high-risk had a 2-year overall survival of $46 \%$ and $2 \%$, respectively.

When investigated further, this biomarker panel proved to have high discriminatory power and, importantly, was able to eliminate the effects of treatment in the model used, implying that treatments that improve the outcomes of patients (in this case abiraterone) modify the numbers of patients who are considered high-risk or low-risk.

The biomarker panel tested in this study is an effective tool for discrimination between high-risk and low-risk patients with CRPC, and satisfied the Prentice biomarker classification criteria.

The authors suggest that these results, from a phase III clinical trial population, are an encouraging step towards clinical implementation of biomarkers for the prognosis of prostate cancer, although further testing, in independent populations, is required.

\section{Peter Sidaway}

This article has also been published in Nat. Rev. Clin. Oncol. (doi:10.1038/nrclinonc.2015.68)

Original article Scher, H. I. et al. Circulating tumor cell biomarker panel as an individual-level surrogate for survival in metastatic castration-resistant prostate cancer. J. Clin. Oncol. doi:10.1200/JC0.2014.55.3487 
ERRATUM

Prostate cancer: New biomarker panel prognosticates patient survival Peter Sidaway

Nat. Rev. Urol. 12, 241 (2015); doi:10.1038/nrurol.2015.154

In the citation line of the originally published article the original doi was incorrect.

This error has been corrected for the HTML and PDF versions of the article. 